\section{Managing the high: developing legislation and detection methods for cannabis impairment}

\author{
Thomas R. Arkell, Amie C. Hayley and Luke A. Downey
}

We commend Ramaekers and colleagues for their timely and comprehensive Review exploring the determinants of neurocognition during acute cannabis exposure (Ramaekers, J. G., Mason, N. L., Kloft, L. \& Theunissen, E. L. The why behind the high: determinants of neurocognition during acute cannabis exposure. Nat. Rev. Neurosci. 22, 439-454 (2021)) ${ }^{1}$. In their article, the authors argue that modulation of activity in the mesocorticolimbic and salience networks may underlie the prototypical acute neurocognitive effects of cannabis, including altered attention and psychomotor function, reduced impulse control, impaired memory and learning, and changes in consciousness. It is well established that the magnitude and duration of these effects can vary substantially among individuals ${ }^{2}$, and the Review by Ramaekers and colleagues further highlights the importance of understanding the determinants of these effects, including genetic factors, age, sex, tolerance, as well as the underlying condition in the case of patients using cannabis therapeutically. Developing knowledge in this area will have a considerable impact on how we manage and offset potential risks for policy makers and consumers, both now and in the future ${ }^{3}$. Here, we would like to briefly address the key question of how we use this information to guide public health policy and treatment involving medical cannabis in a meaningful way.

One way in which we can guide future health policy decisions with due consideration of the biological, pharmacological and behavioural factors that can modulate acute cannabis effects is by setting uniform standards around $\Delta^{9}$-tetrahydrocannabinol (THC) dosing. For example, the implementation of a standard unit of THC (5 mg), much like the use of a 'standard drink' for alcohol, was recently proposed ${ }^{4}$. Not only would the introduction of such standards facilitate meaningful comparisons across studies involving different types of cannabis and varying methods of administration, but it would also support informed decision-making among consumers.

To address the urgent and complex challenge of cannabis-impaired driving that was raised in the Review and elsewhere ${ }^{1,5,6}$, we have proposed an integrated biometric framework for detecting impairment based on established standards of altered neurobehaviour due to acute cannabis intoxication (and other psychoactive substances) ${ }^{7}$. Driver-monitoring systems that are designed to monitor and detect driver state (for example, using gaze monitoring or eye-closure metrics) offer an objective means of indexing impairment associated with driving under the influence of cannabis. These sophisticated vehicle safety systems are capable of scrutinizing and indexing eye movement behaviour and other physiological markers known to influence driving and driver behaviour. If a reliable physiological signal from the eyes could be identified, roadside assessment of ocular functioning could be utilized in place of the assessment of THC concentrations in biological matrices, which correlate poorly with magnitude of neurocognitive impairment ${ }^{8}$ and do not reliably indicate intoxication and/or driving impairment ${ }^{9,10}$.

Apropos of the ongoing shifts in cannabis legislation and increasing global cannabis exposure noted in the Review, we agree that relaxation of restrictions around the use of Good Manufacturing Practice (GMP)-grade cannabis for clinical trials will significantly advance scientific and medical research in this area. Not only are there few GMP-grade cannabis suppliers globally, but this standard is not equivalently applied to products that are currently available to consumers. We also welcome the recent decision by the US Drug
Enforcement Administration (DEA) ${ }^{11}$ to increase opportunities for such research in the USA by removing the stringent restriction requiring researchers to use cannabis from a single domestic source contracted by the National Institute on Drug Abuse.

There is a reply to this letter by Ramaekers, J. G., Mason, N. L., Kloft, L. \& Theunissen, E. L. Reply to: Managing the high: developing legislation and detection methods for cannabis impairment. Nat. Rev. Neurosci. https://doi.org/10.1038/s41583-021-00501-4 (2021).

Thomas R. Arkell, Amie C. Hayley and Luke A. Downey (D) ${ }^{凶}$

Centre for Human Psychopharmacology, Swinburne University, Melbourne, Victoria, Australia.

凶e-mail: Idowney@swin.edu.au https://doi.org/10.1038/s41583-021-00500-5

1. Ramaekers, J. G., Mason, N. L., Kloft, L. \& Theunissen, E. L. The why behind the high: determinants of neurocognition during acute cannabis exposure. Nat. Rev. Neurosci. 22, 439-454 (2021).

2. McCartney, D., Arkell, T. R., Irwin, C. \& McGregor, I. S Determining the magnitude and duration of acute $\Delta(9)$-tetrahydrocannabinol ( $\Delta(9)$ - $\mathrm{THC}$ )-induced driving and cognitive impairment: A systematic and metaanalytic review. Neurosci. Biobehav. Rev. 126, 175-193 (2021)

3. Downey, L. A. \& Hayley, A. C. Prescription medication use and crash risk: taking responsibility for a new global challenge. Lancet Public Health 6, e349-e350 (2021).

4. Freeman, T. P. \& Lorenzetti, V. 'Standard THC units': a proposal to standardize dose across all cannabis products and methods of administration. Addiction 115, 1207-1216 (2020).

5. Arkell, T. R. et al. Effect of Cannabidiol and Delta9Tetrahydrocannabinol on Driving Performance: A Randomized Clinical Trial. JAMA 324, 2177-2186 (2020).

6. Ramaekers, J. G. Driving under the influence of cannabis: an increasing public health concern. JAMA 319, 1433-1434 (2018)

7. Hayley, A. C., Shiferaw, B. \& Downey, L. A. Amphetamine-induced alteration to gaze parameters: A novel conceptual pathway and implications for naturalistic behavior. Prog. Neurobiol. 199, 101929 (2021).

8. Arkell, T. R., Spindle, T. R., Kevin, R. C., Vandrey, R. $\&$ McGregor, I. S. The failings of per se limits to detect cannabis-induced driving impairment: Results from a simulated driving study. Traffic Inj. Prev. 22, 102-107 (2021).

9. Logan, B., Kacinko, S. L. \& Beirness, D. J. An evaluation of data from drivers arrested for driving under the influence in relation to per se limits for cannabis. $A A A$ Foundation https://aaafoundation.org/wp-content/ uploads/2017/12/EvaluationOfDriversInRelationTo PerSeReport.pdf (2016)

10. Huestis, M. A. \& Smith, M. L. Cannabinoid markers in biological fluids and tissues: revealing intake. Trends Mol. Med. 24, 156-172 (2018).

11. Drug Enforcement Administration, Department of Justice. Controls To Enhance the Cultivation of Marihuana for Research in the United States. Federal Register https://www.federalregister.gov/d/ 2020-05796 (2020)

Competing interests

The authors declare no competing interests. 